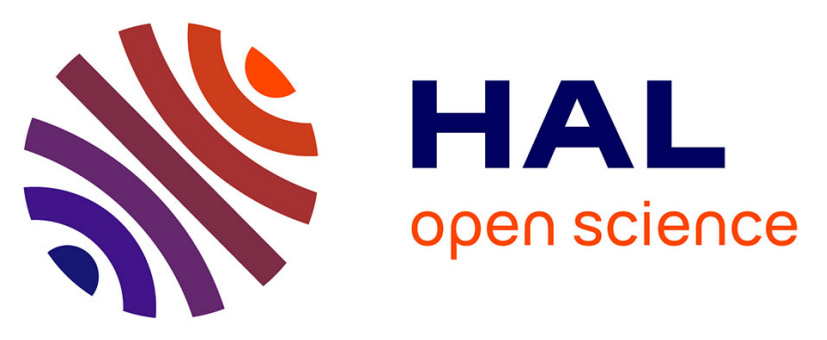

\title{
Insulated Molecular Wires: Sheathing Semiconducting Polymers with Organic Nanotubes through Heterogeneous Nucleation
}

Gijo Raj, Athmane Boulaoued, Johann Lacava, Laure Biniek, Philippe J. Mesini, Martin Brinkmann, Jérôme Faure-Vincent, Jean-Michel Guenet

\section{To cite this version:}

Gijo Raj, Athmane Boulaoued, Johann Lacava, Laure Biniek, Philippe J. Mesini, et al.. Insulated Molecular Wires: Sheathing Semiconducting Polymers with Organic Nanotubes through Heterogeneous Nucleation. Advanced Electronic Materials, 2017, 3 (1), pp.1600370. 10.1002/aelm.201600370 . hal-01502559

\section{HAL Id: hal-01502559 \\ https://hal.science/hal-01502559}

Submitted on 17 Jan 2022

HAL is a multi-disciplinary open access archive for the deposit and dissemination of scientific research documents, whether they are published or not. The documents may come from teaching and research institutions in France or abroad, or from public or private research centers.
L'archive ouverte pluridisciplinaire HAL, est destinée au dépôt et à la diffusion de documents scientifiques de niveau recherche, publiés ou non, émanant des établissements d'enseignement et de recherche français ou étrangers, des laboratoires publics ou privés. 
G. Raj ${ }^{1,2,3}$, A. Boulaoued ${ }^{1,2,3,4}$, J. Lacava ${ }^{4}$, L. Biniek ${ }^{4}$, P.J. Mésini ${ }^{4}$, M. Brinkmann ${ }^{4}$, J. Faure-Vincent ${ }^{1,2,3 *}$, J-M. Guenet $^{4 *}$

1,2,3Univ. Grenoble Alpes, INAC-SyMMES, F-38000 Grenoble, France

${ }^{1,2,3}$ CEA, INAC-SyMMES, F-38000 Grenoble, France

${ }^{1,2,3}$ CNRS, SyMMES, F-38000 Grenoble, France

${ }^{4}$ Institut Charles Sadron, CNRS-Université de Strasbourg, 23 rue du Loess 67034 STRASBOURG cedex02, France

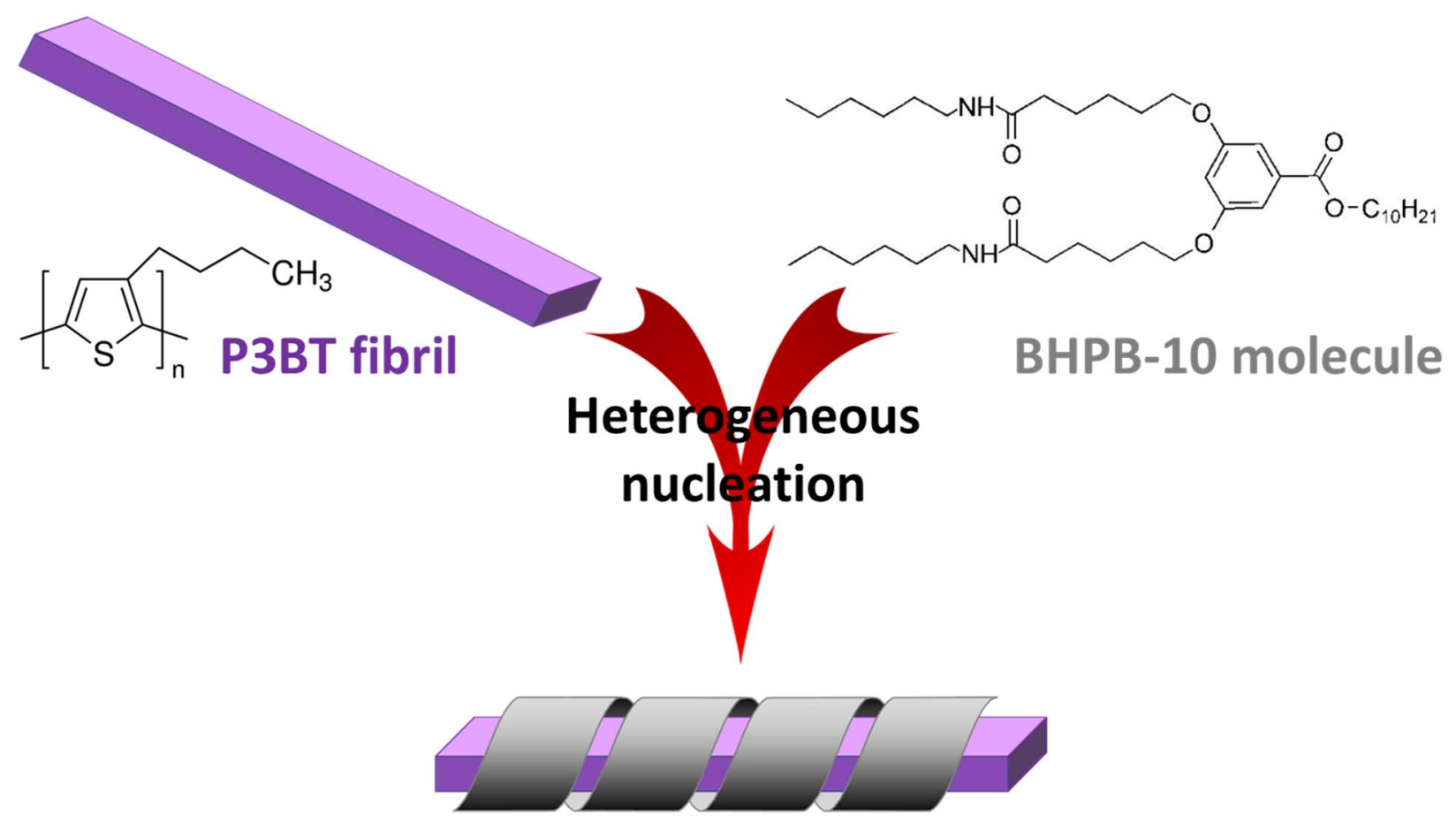

\section{Insulated Molecular Wire}

Keywords : molecular wires, conducting polymer, nanotubes, sheathing, heterogeneous nucleation,

\section{Abstract}

Insulated molecular wires formed from organic molecules may have promising applications in organic and flexible electronic devices. Here, we provide compelling evidence of the formation of insulated Molecular Wires by sheathing conducting regioregular Poly(3-butylthiophene-2,5-diyl) (P3BT) fibrils with insulating 
nanotubes from 3,5-bis-(5-hexylcarbamoylpentyloxy)-benzoic acid decyl ester molecules through a nucleation and growth process. For dilute systems, conducting Atomic Force Microscopy (C-AFM) together with Force-distance curves and Current-Voltage spectroscopy are concomitantly performed to sense current from the topmost surface to the core of the composite fibrils at controlled tip indentation depths. Results show that current is sensed only when the core of the nanocomposite fibrils is reached which indicates the presence of an insulating layer around the semi-conducting P3BT fibrils. The existence of this molecular nanocomposite is further supported by neutron scattering experiments carried out on more concentrated systems at different temperatures.

\section{Introduction}

One-dimensional (1D) nanomaterials, such as nanowires, nanorods and nanotubes, show unique and fascinating properties. In order to enhance some of their properties, to combine two complementary functional materials or to increase their stability or durability, coaxial hybrid nanomaterials have been intensively studied ${ }^{1,2}$. This class of materials have received growing interest owing to their essential implication in both fundamental investigation of physical phenomena (such as charge carrier transport and spin propagation) and practical application development (electronic and optoelectronic devices) ${ }^{2-6}$. Methods using top-down (microfabrication) or bottom-up (e.g. Chemical Vapour Deposition or Atomic Layer Deposition) processes have yielded inorganic core/shell nanowires used in photovoltaic devices ${ }^{2,3,5}$, light emitting diodes ${ }^{7}$, biological and chemical species detection ${ }^{8}$. However, such approaches require extremely stringent methodology and does not lead to nanowires with diameter less than a few tens of $\mathrm{nm}^{3,9,10}$ (top-down) or require precursor materials or high temperature process ${ }^{5}$ (bottom-up). To overcome these limitations, intense research was focused onto a new class of materials designated as Insulated Molecular Wires (IMW) ${ }^{11}$. IMW or organic coaxial nanowires have emerged as alternative materials that can be prepared by chemical synthesis ${ }^{12-15}$, surface functionalization ${ }^{16}$ or non-covalent self-assembly ${ }^{16-21}$. The latter is clearly highly promising as it is the easiest way to build up complex architectures, provided that supramolecular assembly is mastered ${ }^{22}$. In this article, we describe the making and characterization of quasi-1D insulated nanowires made from a pi-conjugated polymer and a small nanotube-forming organic 
molecule. In contrast to the IMW obtained by complex chemical processes, the non-covalent assembly strategy followed here is based only on two physical processes, heterogeneous nucleation and thermoreversible gelation. The proof of concept for making materials through this process has been recently obtained with a non-conducting polymer, isotactic polystyrene, sheathed by the same self-assembling molecule used here ${ }^{23}$. In the present work, nanocomposite fibrils are made up of a Poly(3-butylthiophene2,5-diyl) (P3BT) $\pi$-conjugated polymer-sheathed by a nanotube of a self-assembling molecule, namely 3,5bis-(5-hexylcarbamoylpentyloxy)-benzoic acid decyl ester (BHPB-10) ${ }^{24}$. P3BT was chosen because it belongs to the poly(3-alkylthiophene) family which is well-known in the organic electronics ${ }^{25}$ and for which fibrils has already shown interesting properties ${ }^{26,27}$. The length of our composites fibers is up to several microns, one order of magnitude longer than the previously reported IMWs obtained from solution process ${ }^{11,13,14,28}$. Our fibers have been characterized by Small Angle Neutron Scattering (SANS) as well as Atomic Force Microscopy (AFM). Current spectroscopy has been carried out by Conducting AFM (C-AFM) to probe the conducting core/insulating shell nature of our material. In addition, cross-talk effects between the nanocomposite fibers are absent on micrometer scale, a pre-requisite to their use as IMWs in organic electronics.

\section{The systems}

The different systems observed by TEM are shown in Fig. 1. P3BT forms fibrils of square cross-section with dimensions within 5 to $15 \mathrm{~nm}$; BHPB-10 forms ringlets that are locally nanotubes with outer diameter 12 $\mathrm{nm}$ and inner diameter $9 \mathrm{~nm}$. If a homogeneous mixture of P3BT and BHPB-10 is prepared by heating above $100^{\circ} \mathrm{C}$ and then quenched, one can observe that BHPB-10 nucleates the growth of P3BT fibrils. Conversely, when the protocol described in Fig. 1 is implemented then the hybrid system appears quite different. Mixing the two components above $100^{\circ} \mathrm{C}$ so as to obtain a homogeneous solution, and then quenching to $0^{\circ} \mathrm{C}$ entails the nucleation of the P3BT fibrils by the BHPB-10 nanotubes. This is so because the formation kinetics of P3BT crystals is much slower than the formation of nanotubes. Conversely, when the BHPB-10 in solution in trans-decahydronaphthalene (BHPB-10 SOL) is added at $60^{\circ} \mathrm{C}$ to a suspension of fibrils and then cooled to $0^{\circ} \mathrm{C}$, the hybrid system is produced. 

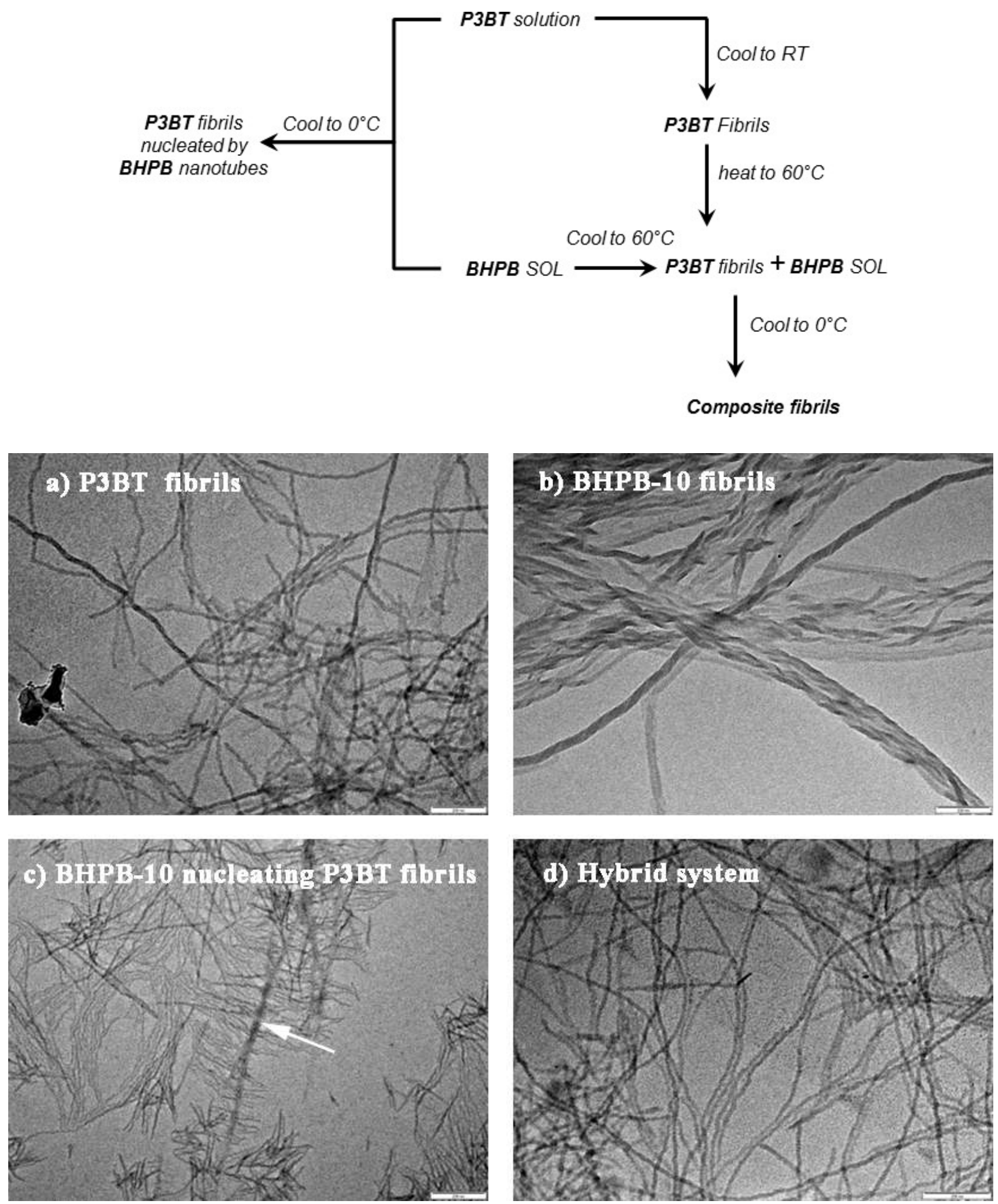

Figure 1: The preparation protocol, top; bottom TEM pictures obtained on: a) purified P3BT fibrils prepared in trans-decahydronaphthalene at room temperature; b) BHPB-10 ringlets produced by quenching at $0^{\circ} \mathrm{C}$ solutions in trans-decahydronaphthalene; c) P3BT fibrils nucleated onto BHPB-10 nanotubes (arrow) by cooling a homogeneous mixture $\left.\left(0.05 \times 10^{2} \mathrm{~g} / \mathrm{cm}^{3} \mathrm{P} 3 \mathrm{BT}+0.01 \times 10^{2} \mathrm{~g} / \mathrm{cm}^{3} \mathrm{BHPB}-10\right) ; \mathrm{d}\right)$ hybrid system obtained by mixing P3BT fibrils grown beforehand with BHPB- 10 SOL at $60^{\circ} \mathrm{C}$ and then quench to $0^{\circ} \mathrm{C}$. White scale bar $=200 \mathrm{~nm}$. Note that the systems are diluted to 10 to 100 times to allow for TEM observations. 


\section{Current spectroscopy using C-AFM technique at controlled indentation depths}

Conducting Atomic Force Microscopy (C-AFM) has been used to probe the electrical properties of the molecular nanostructures under investigation, namely, P3BT fibrils, BHPB-10 fibrils and the hybrid nanomaterial. Current-Voltage (I-V) spectroscopy on C-AFM setups is a tool to have access to the electronic nature of the investigated material deposited on a conducting substrate ${ }^{29}:$ no collected current if the material is electrically insulating, non-linear I-V response if the material is semi-conducting and a linear I-V response if the material is conducting. Force-distance (FZ) experiments are generally used to measure intrinsic properties such as stiffness, hydrophobicity or surface charge density of a given material through the adhesion forces felt by the AFM tip upon retraction ${ }^{30,31}$. The precise vertical Z-displacement of the piezoelectric scanner allows controlling the tip penetration with respect to the material surface. In the present work, FZ curves and I-V spectroscopy are concomitantly used to sense current from the topmost surface of the fiber as well as from the fiber core at controlled indentation depths (figure 2A).

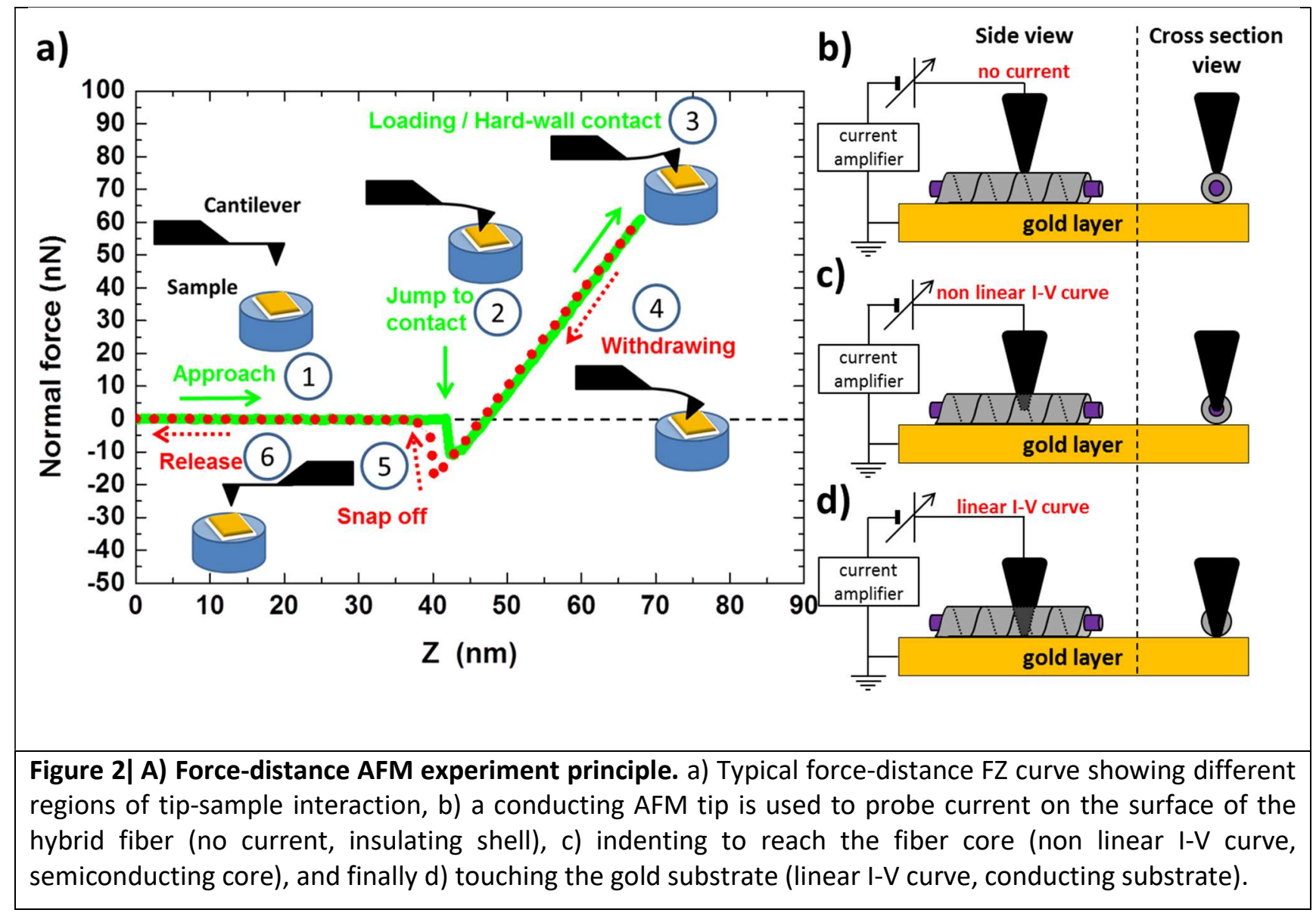


The organic material is spin-coated on an $80 \mathrm{~nm}$ thick gold layer which is grounded using a conductive silver paint. The current is probed by means of a conducting platinum silicide (PtSi) AFM tip (Nanosensors, model: PtSi-FM) as a second terminal. I-V curves recorded on a bare grounded gold electrode give a linear relation with a steep slope as expected in the case of a conducting material (Supplementary Fig. 2).




The principle of the FZ approach is illustrated on Fig. 2A: the force felt by the cantilever is recorded as the function of the piezoelectric vertical displacement $Z$ of the sample holder. When the tip is far from the surface, the force is zero. Upon further approach, a jump to contact occurs when a negative deflection is seen in the FZ curve arising from the attraction of the tip to the surface on account of long-range attractive forces. On further loading the force becomes proportional to the displacement while the penetration depth of the tip into the material depends on the sample stiffness. The $Z$ distance after the 'jump to contact' is the sum of cantilever deflection and the tip penetration/indentation depth ${ }^{30}$. A reference sample made of freshly cleaved mica is used to calculate accurately the penetration depth $\Delta Z\left(=Z_{\text {material }}-Z_{\text {mica }}\right)$. Upon tip withdrawing ( $Z$ decreasing), the sharp decrease to zero force corresponds to the contact snap off. The electronic properties of P3BT, BHPB-10, and the hybrid fibers are characterized by I-V curves registered at several tip depths into the different organic materials. Experimental details can be found in the supplementary material.

On pure P3BT fibrils, current is measured instantly at the jump to contact of the tip with the fiber surface. Non-linear I-V curves indicate the semi-conducting nature of pure P3BT as expected (Supplementary Fig.3). The current saturation level (at $100 \mathrm{nA}$ ) is reached when the tip reaches the gold layer, which corresponds to $\Delta \mathrm{Z}=4.5 \mathrm{~nm}$. This value is in excellent agreement with the height of the fiber derived from standard AFM cross-section analysis $(4.2 \mathrm{~nm})$.

In the case of pure BHPB-10 nanotubes, no current is sensed upon jump into contact or further indentation, confirming its insulating nature (Supplementary Fig. 4), until the tip touches the grounded electrode, leading to current saturation.

For the composite fibrils, no current is sensed upon jump to contact (Fig. 2B) and down to a distance of 6.6 $\mathrm{nm}$ from the top surface of the fiber (see Fig. $2 \mathrm{~B}(\mathrm{c})$ ). Thus the conducting AFM tip is first sensing a layer of insulating material on the top of the fiber surface, as illustrated on Fig. $2 A(b)$. A small value of current is being sensed when tip penetrates $7.8 \mathrm{~nm}$ into the composite fiber (Fig. 2B (d). Here the AFM tip is just approaching the conducting part of the composite fiber. A further increment in the penetration depth results in a clear jump of current $(\approx 10 \mathrm{nA}$ for $1 \mathrm{~V})$ as the tip establishes subsequent contact with the semi- 
conducting domain (in our case P3BT) as ascertained by the non-linear variation of the I-V curve on Fig. 2 $\mathrm{B} €$ and as illustrated on Fig. $2 \mathrm{~A}$ (c). Allowing the tip to penetrate deeper into the fibril entails a current saturation which corresponds to the reaching of the gold grounded electrode (Fig. 2 A(d)). This experiment reveals that the I-V response of a P3BT-BHPB-10 composite fiber is very different when compared to that of a pure P3BT fiber, in the later where current is sensed at the very instant when the tip is in contact with the fiber. The fact that current is sensed only from the core of the fiber and not from the topmost layers is indeed direct evidence to the core-shell structure composed of a conducting P3BT core sheathed by an insulating BHPB-10.

Here, the question may arise as to whether there is a real sheathing through a heterogeneous nucleation process or simply P3BT fibrils being accidentally covered by BHPB-10 material. To test this point, films were prepared by first spin coating P3BT fibrils on a gold electrode, followed by the spin coating of BHPB-10 material, and drying under vacuum. Cross-section analysis of the sequentially deposited film show two types of fibers with $5 \mathrm{~nm}$ and $30 \mathrm{~nm}$ heights that correspond to the heights of P3BT fibrils and BHPB-10 nanotubes, respectively. This clearly indicates that P3BT and BHPB-10 fibrils are well-separated entities under these conditions. FZ and I-V measurements on BHPB-10 bundles show that no current is sensed at the jump to contact region. When the tip traveled a distance equivalent to the BHPB-10 fiber bundle height, current is sensed from underlying P3BT/Au electrode (See Supplementary Fig. 4).

Further investigations into the electrical properties of the fibers were performed by mapping current flowing across ground and isolated Au electrodes separated by an insulating $\left(\mathrm{SiO}_{2}\right) 5 \mu \mathrm{m}$-wide channel. The idea is to highlight the proof of concept of these composite fibers as IMWs: conduction of current by the inner part along the fiber axis and electrical insulation by the outer shell. On this purpose, we used contact mode C-AFM, where current maps are generated concomitantly with topography images. A Pt/Ir coated tip (Nanosensors, model: PPP-CONTPt) is scanned over the sample surface with an applied bias voltage (fixed at $1 \mathrm{~V}$ ) between the ground electrode and the tip end (acting as the second terminal): for each pixel of the C-AFM image the value of the collected current at this position is recorded. The $1 \mathrm{~V}$ voltage was chosen to unambiguously distinguish between the insulating or conducting nature of the materials under consideration. The principle of the experiment is drawn in Fig. $2 \mathrm{C}(\mathrm{a})$ which represents the five possible 
AFM tip positions in the case of the hybrid fibers: i) directly in contact with the grounded gold electrode (current flows), ii) on top of a hybrid fiber on the grounded electrode (no current, configuration similar to Fig. 2-b), iii) on top of a hybrid fiber in the insulating $\mathrm{SiO}_{2}$ channel (no current), iv) on top of a hybrid fiber on the isolated electrode (no current) and v) directly in contact with the isolated gold electrode (current flows thanks to the hybrid fibers acting as IMWs). Current map of the hybrid fibers is shown on Fig. 2 C, while that of the pure P3BT and pure BHPB-10 fibers can be found in the Supplementary Materials (Supplementary Fig. 5). In the case of hybrid fibers, current appears on both grounded (case i) and isolated (case v) electrodes revealing that these electrodes are electrically connected. In addition, i) the channel area is devoid of any current despite topography image showing fibers across the channel and ii) these hybrid fibers appear dark (i.e. no current) on the grounded and isolated electrodes: these two points clearly point out that the hybrid fibers have an insulating outer part, which impedes the current flow (cases ii, iii and iv). Though current appears at the isolated electrode, the absence of current in the channel is at variance with the high current value observed in the channel for pure P3BT fibrils. Indeed, when pure P3BT fibrils are deposited, current appears with the same intensity on both electrodes as well as across the insulating channel between them, indicating the conducting nature of P3BT fibers (Supplementary Fig. 5a). In the case of pure BHPB-10 fibers, current appears only on the ground electrode while no current is sensed on the channel or at the isolated electrode. It is interesting to note here that areas corresponding to BHPB10 fibers on the ground electrode appear dark in the current map in agreement with the insulating character of BHPB-10 (See Supplementary Fig. 5b). These C-AFM results confirm the sheathing of BHPB-10 over P3BT that renders the composite fiber insulating properties perpendicular to the fiber axis (absence of channel current, hybrid fibers appearing dark even on the grounded electrode), and conducting properties in the co-axial direction (current appears on the isolated electrode). From these experiments, it is also clear that the electrical cross-talk is prevented even on large fiber bundles and over large distances ( $5 \mu \mathrm{m}$-wide channel). This confirms the use of our composite material as efficient IMWs. 


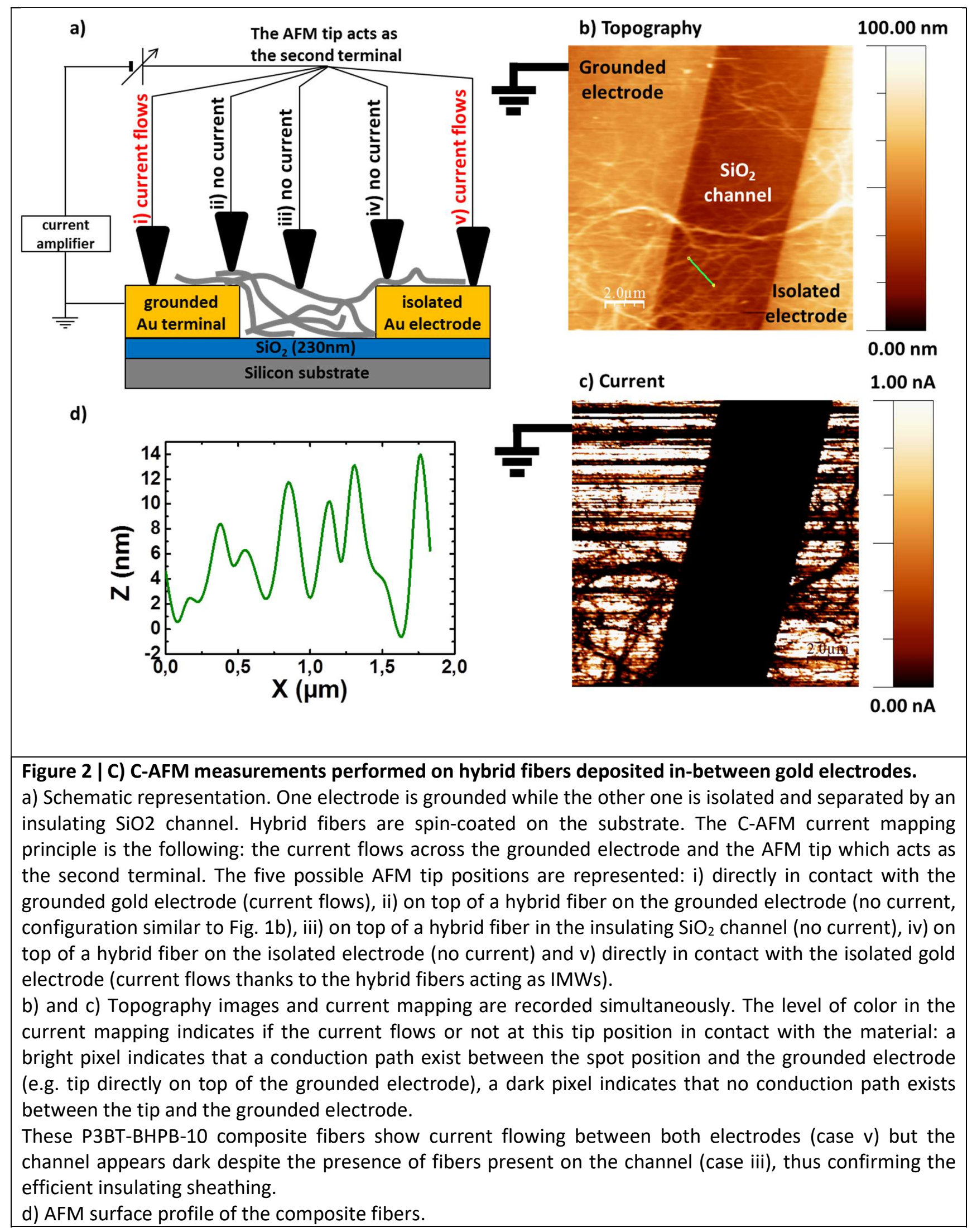




\section{Small-Angle Neutron Scattering experiments}

The small-angle neutron scattering were carried out on three samples: BHPB-10/transdecahydronaphthaleneD, $C_{\mathrm{BHPB}-10}=0.01 \mathrm{~g} / \mathrm{cm}^{3}, \mathrm{P} 3 \mathrm{BT} /$ trans-decahydronaphthaleneD, $\mathrm{C}_{\mathrm{P} 3 \mathrm{~B}}=0.01 \mathrm{~g} / \mathrm{cm}^{3}$, and the ternary system BHPB-10/P3BT/trans-decahydronaphthaleneD, $\mathrm{C}_{\mathrm{BHPB}-10}=0.01 \mathrm{~g} / \mathrm{cm}^{3}+\mathrm{C}_{\mathrm{P} 3 \mathrm{~B}}=0.01 \mathrm{~g} / \mathrm{cm}^{3}$. These systems were studied at $25^{\circ} \mathrm{C}, 47^{\circ} \mathrm{C}$, and $60^{\circ} \mathrm{C}$, respectively. The results are presented in Fig. 3 by means of a Kratky-representation ( $q^{2} I(q)$ vs $\left.q\right)$. The scattering curve of BHPB-10 can be well-fitted with the scattering function of a hollow cylinder:

$$
\mathrm{I}(\mathrm{qr})=\frac{\pi \mu_{\mathrm{L}}}{\mathrm{q}}\left[\frac{2}{\left(1-\gamma^{2}\right) \mathrm{qr}} \times\left(\mathrm{J}_{1}(\mathrm{qr})-\gamma \mathrm{J}_{1}(\gamma \mathrm{qr})\right]^{2}\right.
$$

where $r$ is the outer radius of the cylinder, $\nu$ the ratio between the inner and the outer radii, and $\mu_{\mathrm{L}}$ the mass per unit length.

The fit yields $r_{\text {out }}=12 \mathrm{~nm}$ and $\nu=0.75$, results in good agreement with previous finding ${ }^{23,24}$ on this molecule. As P3BT forms fibrils with rectangular cross-sections, a good fit can be obtained for the P3BT scattering curve by considering straight ribbons whose theoretical form factor reads:

$$
\varphi(\mathrm{qr})=\frac{2}{\pi} \int_{0}^{\pi / 2}\left[\frac{\operatorname{sinqa} / 2 \cos }{\mathrm{qa} / 2 \cos \theta} \times \frac{\operatorname{sinqb} / 2 \sin \theta}{\mathrm{qb} / 2 \sin \theta}\right]^{2} \sin \theta \mathrm{d} \theta
$$

The fit presented in Fig. $5 a$ corresponds to $a=4 \mathrm{~nm}$ and $\mathrm{b}=14 \mathrm{~nm}$. The diagonal of the cross-section amounts therefore to about $15 \mathrm{~nm}$. These values correspond to values that we have observed by TEM and AFM]. A better fit could certainly be obtained by taking into account a slight cross-sectional polydispersity but this is not the direct concern of this paper.

As highlighted in Fig. 3, the scattering curve for the ternary system, $I_{B t e r n a r y}^{\text {exp }}$, is not the sum of the scattering curves determined for the binary systems as it should have been if no particular interaction were taking place. It also shows oscillations, yet the maximum at $q=0.31 \mathrm{~nm}^{-1}$ observed for this sum is shifted to $\mathrm{q}=0.25 \mathrm{~nm}^{-1}$, and the magnitude of the intensities do not match. Either intramolecular terms have to be considered or the occurrence of a hybrid system involving the P3BT fibrils and the BHPB-10 nanotubes ought to be considered. As will be highlighted below, the results obtained at $60^{\circ} \mathrm{C}$ definitely allows one to disregard intermolecular terms. 

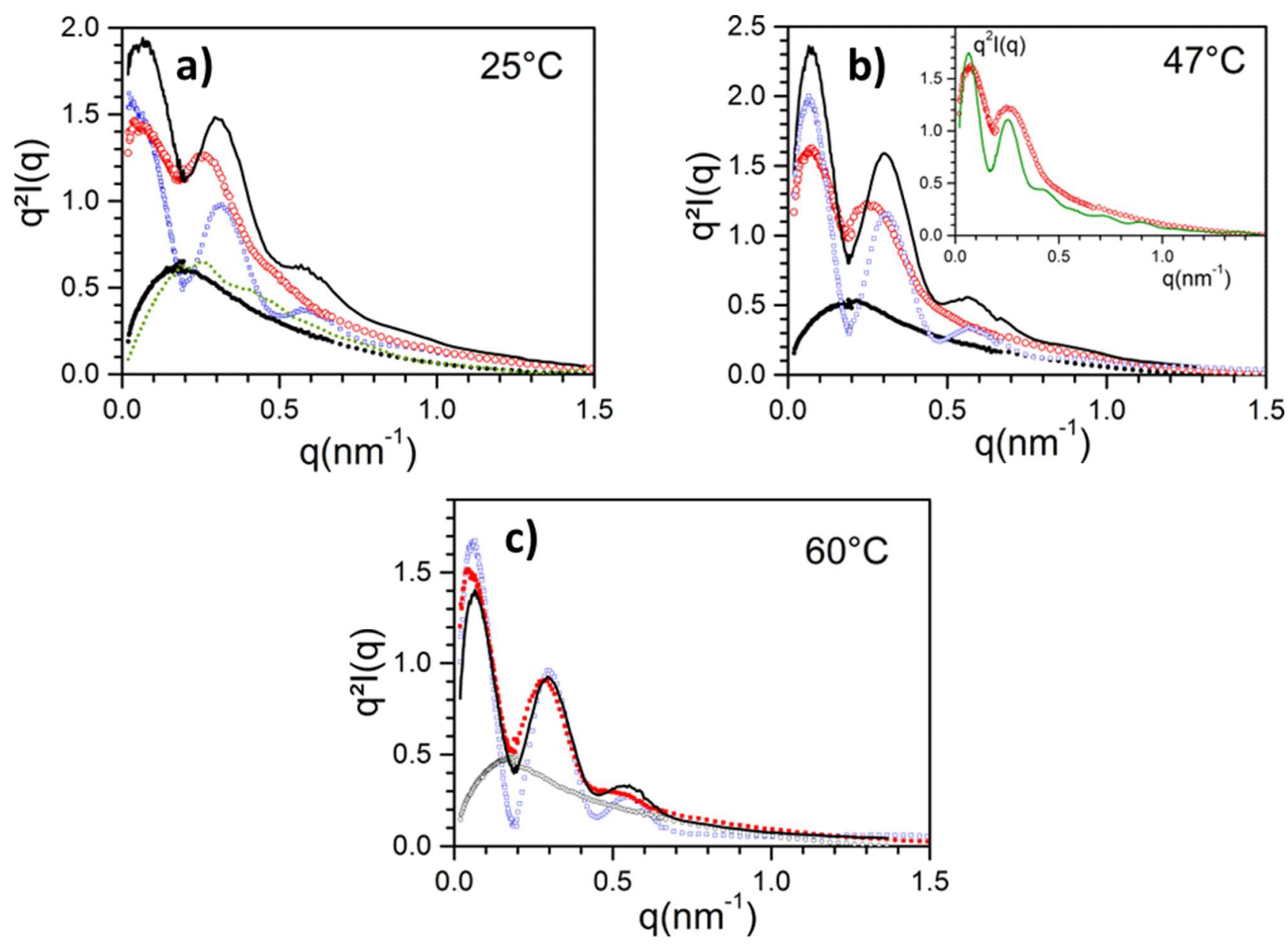

Figure 3 | Small-angle scattering curves plotted by means of a Kratky-plot ( $q^{2} I(q)$ vs $\left.q\right)$ for the binary system P3BT/trans-decahydronapthalene gels, $\mathrm{C}_{\text {Рзвт }}=0.01 \mathrm{~g} / \mathrm{cm}^{3}$ (black dots); the binary system BHPB$10 /$ trans-decahydronaphthalene gels, $C_{B H P B-10}=0.01 \mathrm{~g} / \mathrm{cm}^{3}$ (blue open squares); the ternary system P3BT/BHPB-10/trans-decahydronaphthalene, $\mathrm{C}_{\text {ВнРВ-10 }}=0.01 \mathrm{~g} / \mathrm{cm}^{3}+\mathrm{C}_{\text {Рзвт }}=0.01 \mathrm{~g} / \mathrm{cm}^{3}$ (red open circles); the solid curve stands for the sum of the intensities of each binary system, except at $60^{\circ} \mathrm{C}$ where it represents $70 \%$ of this sum. a) $\mathrm{T}=25^{\circ} \mathrm{C} \mathrm{b}$ ) $\mathrm{T}=47^{\circ} \mathrm{C}$, inset shows the best fit in solid green curve (see text for details)and c) $\mathrm{T}=60^{\circ} \mathrm{C}$.

The same fits basically work at $47^{\circ} \mathrm{C}$ although the scattered intensity of the P3BT in the binary mixture has slightly decreased; possibly due to the melting of some of the fibrils and thermal expansion of the solvent (Fig. 3b).

The results obtained at $60^{\circ} \mathrm{C}$ rather support the existence of a hybrid system against the existence of intermolecular terms (Fig. 3c). As can be seen, the scattered intensity is this time the sum of the scattered intensity of each component in the binary systems scaled by a factor 0.7 . This disregards the effect of intermolecular terms for the lower temperatures in the explored q-range. Clearly, a fraction of the material has vanished that is neither pure P3BT fibrils nor pure BHPB-10 nanotubes. Note that melted material, 
particularly BHPB-10, scatters very little compared to the polymer fibrils and the nanotubes so that its contribution to the intensity can be ignored to a good approximation.

If one contemplates the possible occurrence of a hybrid system where BHPB-10 nanotubes sheathe P3BT fibrils, then this can be modelled with two concentric cylinders assuming in a first approximation a cylinderlike structure for the P3BT fibrils. In view of the lower scattering of P3BT fibrils with respect to BHPB-10's this is a very good approximation (see supp. Information). In other word the scattering is dominated by the BHPB-10 moiety.

This approach is definitely based on the C-AFM outcome that definitely allows one to narrow down the possible library of models. One has to keep in mind that each of these concentric cylinders possesses a different neutron contrast factors in trans-decahydronaphthalene (see supplementary information) so that the theoretical expression for the scattered intensity reads ${ }^{32}$ :

$$
\mathrm{I}_{\text {hybrid }}^{\text {theo }}(\mathrm{q}) \sim \frac{\pi \mu_{\mathrm{LHyb}}}{\mathrm{q}}\left[\frac{2 \gamma \mathrm{A}_{\text {in }}}{\mathrm{A}_{\mathrm{m}} \mathrm{qr}_{\text {out }}} \mathrm{J}_{1}\left(\mathrm{q} \gamma \mathrm{r}_{\text {out }}\right)+\frac{2 \mathrm{~A}_{\text {out }}}{\mathrm{A}_{\mathrm{m}} \mathrm{qr} \text { out }} \times\left(\mathrm{J}_{1}\left(\mathrm{qr}_{\text {out }}\right)-\gamma \mathrm{J}_{1}\left(\gamma q \mathrm{r}_{\text {out }}\right)\right]^{2}\right.
$$

where $A_{\text {in }}$ and $A_{\text {out }}$ are the scattering amplitude of the inner and outer cylinder, and with

$$
\mathrm{A}_{\mathrm{m}}=\gamma^{2} \mathrm{~A}_{\mathrm{in}}+\left(1-\gamma^{2}\right) \mathrm{A}_{\text {out }}
$$

From the results at $60^{\circ} \mathrm{C}$ we infer that about 0.3 of this hybrid material was formed. Therefore, the final expression for the expected intensity at $25^{\circ} \mathrm{C}$ should read:

$$
\mathrm{I}(\mathrm{q})=0.35 \times \mathrm{I}_{\mathrm{P} 3 \mathrm{BT}}^{\exp }(\mathrm{q})+0.35 \times \mathrm{I}_{\mathrm{BHPB}-}^{\exp }(\mathrm{q})+0.3 \times \mathrm{I}_{\text {hybrid }}^{\text {theo }}(\mathrm{q})
$$

where $I_{P 3 B T}^{e x p}$ and $I_{B H P B-10}^{e x p}$ are the experimental intensities measured for the binary systems.

Equal fraction of $\mathrm{P} 3 \mathrm{BT}$ and $\mathrm{BHPB}-10$ are considered since they correspond to the starting material.

In order to match the positions of the first two maximum the outer cylinder should possess an external radius of $r_{\text {out }}=20 \mathrm{~nm}$ and an internal radius, being also the radius of the inner solid cylinder, of $r_{\text {in }}=9 \mathrm{~nm}$ (figure $4 \mathrm{~b}$ inset). This fit should be regarded indicative, although there are not so many possibilities in view of the constraints imposed by the position of the second maximum together with the relative ratios of the first two maxima.

The inner radius, $r_{i n}=9 \mathrm{~nm}$, is consistent with the half-value of the diagonal of the P3BT ribbons $(7.5 \mathrm{~nm})$. Conversely, the outer radius is larger by a factor about 1.6 with respect to the outer radius of the pure 
nanotubes. This gives a layer of about $11 \mathrm{~nm}$, which suggests that approximately 3 layers of BHPB-10 would sheathe the P3BT fibrils since the nanotube thickness is about $3 \mathrm{~nm}$. This value, $11 \mathrm{~nm}$, is consistent with the findings from the C-AFM observations, where the tip has to penetrate by about 8 to $11 \mathrm{~nm}$ to establish contact with the semi-conducting P3BT. Note that the scattering function cannot differentiate between closed nanotubes and helical ribbons at this level of resolution. The sheathing shell may well be made up with one closed nanotube onto which helical ribbons further grow.

Under these conditions a molecular mixture reminiscent of an eutectic compound may be formed which can account the hybrid system melting at a temperature below that the pure BHPB-10 nanotubes ${ }^{23}\left(\mathrm{~T}_{\text {BHPB- }}\right.$ ${ }_{10}=67^{\circ} \mathrm{C}$ for $\left.\mathrm{C}=0.01 \mathrm{~g} / \mathrm{cm}^{3}\right)$ and of the pure P3BT fibrils $\left(T_{\text {P3BT }}=100^{\circ} \mathrm{C}\right)$. The formation of an eutectic system also implies that the degree of sheathing may vary with the concentrations of each species and the composition. Determination of the ternary phase diagram would be a prerequisite for gaining a deeper knowledge on these systems.

\section{Additional information}

See supporting information for details on C-AFM experiments.

\section{Acknowledgement}

Dr. Frédéric Chandezon is acknowledged for fruitful scientific discussions and help with the AFM setup. Neutron scattering experiments have been performed at the Institut Laue-Langevin (ILL) on D11 small-angle camera. We are indebted to M. Schweins, D11 responsible scientist, for his experimental support. Authors acknowledge the Matisse Project (ANR grant 2011 BS08001 02) for financial support. This work has been performed with the use of the Hybriden facility at CEA-Grenoble (France). We also acknowledge funding from the Laboratoire d'excellence LANEF in Grenoble (ANR-10-LABX-51-01). 


\section{References}

1 He, W. et al. Polypyrrole/Silver Coaxial Nanowire Aero-Sponges for Temperature-Independent Stress Sensing and Stress-Triggered Joule Heating. ACS Nano 9, 4244-4251 (2015).

2 Tang, J., Huo, Z., Brittman, S., Gao, H. \& Yang, P. Solution-processed core-shell nanowires for efficient photovoltaic cells. Nat Nano 6, 568-572 (2011).

3 Lauhon, L. J., Gudiksen, M. S., Wang, D. \& Lieber, C. M. Epitaxial core-shell and core-multishell nanowire heterostructures. Nature 420, 57-61 (2002).

$4 \quad$ PramanikS et al. Observation of extremely long spin relaxation times in an organic nanowire spin valve. Nat Nano 2, 216-219 (2007).

5 Tian, B. et al. Coaxial silicon nanowires as solar cells and nanoelectronic power sources. Nature 449, 885-889 (2007).

6 Xia, Y. et al. One-Dimensional Nanostructures: Synthesis, Characterization, and Applications. Advanced Materials 15, 353-389 (2003).

7 Hayden, O., Greytak, A. B. \& Bell, D. C. Core-Shell Nanowire Light-Emitting Diodes. Advanced Materials 17, 701-704 (2005).

8 Peled, A., Pevzner, A., Peretz Soroka, H. \& Patolsky, F. Morphological and chemical stability of silicon nanostructures and their molecular overlayers under physiological conditions: towards longterm implantable nanoelectronic biosensors. Journal of Nanobiotechnology 12, 1-11 (2014).

9 Beltran-Huarac, J. et al. Single-Crystal $\psi$-MnS Nanowires Conformally Coated with Carbon. ACS Applied Materials \& Interfaces 6, 1180-1186 (2014).

10 Jeong, N. et al. Single-Crystal Apatite Nanowires Sheathed in Graphitic Shells: Synthesis, Characterization, and Application. ACS Nano 7, 5711-5723 (2013).

11 Frampton, M. J. \& Anderson, H. L. Insulated Molecular Wires. Angewandte Chemie International Edition 46, 1028-1064 (2007).

12 Cacialli, F. et al. Cyclodextrin-threaded conjugated polyrotaxanes as insulated molecular wires with reduced interstrand interactions. Nat Mater 1, 160-164 (2002).

13 Sugiyasu, K. et al. A Self-Threading Polythiophene: Defect-Free Insulated Molecular Wires Endowed with Long Effective Conjugation Length. Journal of the American Chemical Society 132, 1475414756 (2010).

14 Terao, J. et al. Insulated Molecular Wire with Highly Conductive $\pi$-Conjugated Polymer Core. Journal of the American Chemical Society 131, 18046-18047 (2009).

15 Yamamoto, H. M. et al. Supramolecular Insulating Networks Sheathing Conducting Nanowires Based on Organic Radical Cations. ACS Nano 2, 143-155 (2008).

16 Hirsch, A. Functionalization of Single-Walled Carbon Nanotubes. Angewandte Chemie International Edition 41, 1853-1859 (2002).

17 Chen, C.-A., Yeh, R.-H. \& Lawrence, D. S. Design and Synthesis of a Fluorescent Reporter of Protein Kinase Activity. Journal of the American Chemical Society 124, 3840-3841 (2002).

18 Deria, P. et al. Single-Handed Helical Wrapping of Single-Walled Carbon Nanotubes by Chiral, lonic, Semiconducting Polymers. Journal of the American Chemical Society 135, 16220-16234 (2013).

$19 \mathrm{Li}, \mathrm{L} ., \mathrm{Li}, \mathrm{C}$. Y. \& Ni, C. Polymer Crystallization-Driven, Periodic Patterning on Carbon Nanotubes. Journal of the American Chemical Society 128, 1692-1699 (2006).

20 Star, A. et al. Preparation and Properties of Polymer-Wrapped Single-Walled Carbon Nanotubes. Angewandte Chemie International Edition 40, 1721-1725 (2001).

21 Whitesides, G., Mathias, J. \& Seto, C. Molecular self-assembly and nanochemistry: a chemical strategy for the synthesis of nanostructures. Science 254, 1312-1319 (1991).

22 Bolsée, J.-C., Oosterbaan, W. D., Lutsen, L., Vanderzande, D. \& Manca, J. The Importance of Bridging Points for Charge Transport in Webs of Conjugated Polymer Nanofibers. Advanced Functional Materials 23, 862-869 (2013).

23 Dasgupta, D. et al. Design of hybrid networks by sheathing polymer fibrils with self-assembled nanotubules. Soft Matter 6, 3573-3581 (2010).

24 Díaz, N. et al. Self-Assembled Diamide Nanotubes in Organic Solvents. Angewandte Chemie International Edition 44, 3260-3264 (2005). 
Perepichka, I. F. \& Perepichka, D. F. Handbook of Thiophene-Based Materials: Applications in Organic Electronics and Photonics. (John Wiley \& Sons, Ltd. , 2009).

26 Berson, S., De Bettignies, R., Bailly, S. \& Guillerez, S. Poly(3-hexylthiophene) Fibers for Photovoltaic Applications. Advanced Functional Materials 17, 1377-1384 (2007).

27 Jo, S. B., Lee, W. H., Qiu, L. \& Cho, K. Polymer blends with semiconducting nanowires for organic electronics. Journal of Materials Chemistry 22, 4244-4260 (2012).

28 Masai, H. et al. Enhancement of Phosphorescence and Unimolecular Behavior in the Solid State by Perfect Insulation of Platinum-Acetylide Polymers. Journal of the American Chemical Society 136, 14714-14717 (2014).

29 Hermosa, C. et al. Intrinsic electrical conductivity of nanostructured metal-organic polymer chains. Nat Commun 4, 1709 (2013).

30 Cappella, B. \& Dietler, G. Force-distance curves by atomic force microscopy. Surface Science Reports 34, 1-104 (1999).

31 Heinz, W. F. \& Hoh, J. H. Relative Surface Charge Density Mapping with the Atomic Force Microscope. Biophysical Journal 76, 528-538 (1999).

32 Saiani, A. \& Guenet, J.-M. On the Helical Form in Syndiotactic Poly(methyl methacrylate) Thermoreversible Gels As Revealed by Small-Angle Neutron Scattering. Macromolecules 30, 966972 (1997). 
Insulated molecular wires: sheathing semi-conducting polymers with organic nanotubes through heterogeneous nucleation

G. Raj ${ }^{1,2,3}$, A. Boulaoued ${ }^{1,2,3,4}$, J. Lacava ${ }^{4}$, P.J. Mésini ${ }^{4}$, M. Brinkmann ${ }^{4}$, J. Faure-Vincent ${ }^{1,2,3 *}$, J-M Guenet $^{4 *}$

1,2,3 Univ. Grenoble Alpes, INAC-SyMMES, F-38000 Grenoble, France

${ }^{1,2,3}$ CEA, INAC-SyMMES, F-38000 Grenoble, France

1,2,3 CNRS, SyMMES, F-38000 Grenoble, France

${ }^{4}$ Institut Charles Sadron, CNRS-Université de Strasbourg, 23 rue du Loess 67034 STRASBOURG cedex02, France 


\section{METHODS}

\section{Samples preparation}

The molecule used in this study for its propensity of producing nanotubes in some organic solvents is 3,5-Bis-(5hexylcarbamoylpentyloxy)-benzoic acid decyl ester (abbreviated as BHPB-10 in what follows) whose chemical structure is shown below.

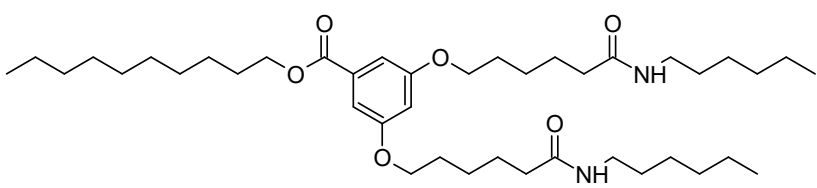

The synthesis of BHPB-10 is described in details in reference ${ }^{24}$.

Poly(3-butylthiophene-2,5-diyl) was purchased from Merck. Its weigh-averaged molecular weight is $\mathrm{Mw}=32$ kDa with a polydispersity $M_{w} / M_{n}=2.0$ and a regioregularity of $77 \%$. The amorphous fraction was removed from the suspensions of crystallized material through successive centrifugations (see supplementary information). Only the P3BT fraction of highest crystallinity was used thereafter.

The hybrid materials prepared through a four-step process (shown in Fig. 1): 1) preparing separately a suspension of P3BT fibrils at room temperature in trans-decahydronaphthalene , 2) preparing separately a homogeneous solution of $\mathrm{BHPB}-10$ in trans-decahydronaphthalene at $\left.\mathrm{T}=70^{\circ} \mathrm{C}, 3\right)$ mixing at $60^{\circ} \mathrm{C}$ the $\mathrm{P} 3 \mathrm{BT}$ suspension and the BHPB-10 solution, 4) quenching the system at $0^{\circ} \mathrm{C}$.

Mixing at $60^{\circ} \mathrm{C}$ allows one to keep a homogeneous solution of BHPB-10 in trans-decahydronaphthalene since nanotube formation occurs only at $\mathrm{T}=45^{\circ} \mathrm{C}$, meanwhile P3BT fibrils are prevented from melting. Quenching at $0^{\circ} \mathrm{C}$ triggers the rapid growth of the BHPB-10 nanotubes.

\section{Experiments}

TEM

After preparation of the samples for the C-AFM experiments, the solutions are further diluted to allow for observation in TEM (typically 10 to 100 times). A drop of the resulting solution is deposited onto a carbon- 
coated copper grid, and the excess of solvent is carefully removed by means of blot-paper. This avoids additional aggregation due to solvent evaporation. TEM was performed in bright field s using a Philips CM12 microscope equipped with a MVIII CCD camera (Soft Imaging System) operating at 120 kV.C-AFM experiments were performed using the Nanotec Cervantes AFM with the Dulcinea controller (Nanotec Electronica, Spain). Images of the fibers deposited on the grounded electrode were acquired in Tapping mode AFM under ambient conditions using PtSi tips (Nanosensors, model: PtSi-FM). Once stable and drift free image of the fibers are recorded, FZ and I-V cures were measured at several position on selected fibers with controlled Z increments. Time to record a single spectral curve is $\sim 2 \mathrm{~s}$ with number of points at least 200 . I-V curves on the grounded electrode give a straight line with a steep slope that is characteristic of a highly conducting material (Supplementary Fig. 1). New tip was used for each set of samples. Tips are then used to perform FZ curves on freshly cleaved mica in order to determine the actual tip penetration distance on the sample.

Small-angle neutron scattering. The experiments were performed on D11 camera located at the Institut LaueLangevin (ILL, Grenoble, France). A wavelength of $\lambda_{m}=0.6 \mathrm{~nm}$ was used with a wavelength distribution characterized by a full width at half maximum of about $10 \%$. A built-in two-dimensional position sensitive detector composed of $64 \times 64$ cells was used (details available on http://www.ill.eu). The sample-detector distance was varied $(3,10,34 \mathrm{~m})$ in order to span a large q-range $(0.05<q(\mathrm{~nm}-1)<2.25)$ with $q=(4 \pi / \lambda) \sin$ $(\vartheta / 2)$, where $\vartheta$ is the scattering angle. The position sensitive detector was calibrated with light water for correcting for cell efficiency and obtaining absolute intensity, $I_{a b s}(q)$. The absolute intensity was finally obtained after the usual signal processing and using $d \Sigma / d \Omega=0.985 \mathrm{~cm}^{-1}$ for the water cross-section as determined experimentally for D11 at $\lambda_{m}=0.6 \mathrm{~nm}$, and the contrast factor for the labelled species.

Per deuterated trans-decahydronaphthalene, $\mathrm{C}_{10} \mathrm{D}_{18}$, purchased from Cambridge Isotopes was used throughout. The scattering amplitudes for P3BT and BHPB-10 in this deuterated solvent used for equation 3 are calculated by means of:

$$
A_{\text {out }}=\left[\frac{A_{B H P B-10}}{v_{B H P B-10}}-\frac{A_{\text {TdecaD }}}{v_{\text {TdecaD }}}\right] \text { and } A_{\text {in }}=\left[\frac{A_{P 3 B T}}{v_{P 3 B T}}-\frac{A_{\text {TdecaD }}}{v_{\text {TdecaD }}}\right]
$$


where $\mathrm{A}$ and $\mathrm{v}$, with the appropriate subscript, are the scattering amplitudes and the molar volumes, respectively, and with $\mathrm{A}_{\mathrm{BHPB}-10} / \mathrm{v}_{\mathrm{BHPB}-10}=0.01096, \mathrm{~A}_{\mathrm{P} 3 \mathrm{~B}} / \mathrm{v}_{\mathrm{P} 3 \mathrm{~B}}=0.0362$, and $\mathrm{A}_{\mathrm{TdecaD}} / \mathrm{v}_{\mathrm{Tdec}}=.0 .1176$. As a reminder, for neutrons $A$ is the sum of the scattering lengths of the atoms constituting the molecule. 


\section{SUPPLEMENTARY FIGURES AND FIGURE CAPTIONS}

\section{Purification of the P3BT samples}

Trans-decahydronaphthalene was purified through long columns of silica gel prior to use.

The as-received P3BT was first washed in a soxhlet by acetone and hexane. Then, the P3BT chains that poorly or even do not crystallize have been removed by successive centrifugations. P3BT is first allowed to crystallize from dilute solutions in trans-decahydronaphthalene $\left(0.075 \times 10^{-2} \mathrm{~g} / \mathrm{cm}^{3}\right)$. Centrifugation separates the P3BT fibrils from chains that have not crystallized, the latter being located in the supernatant. After an ageing time of $24 \mathrm{H}$ to allow for the growth of P3BT fibrils, the samples have undergone three centrifugation processes at 1000 rpm for $2 \mathrm{hrs}$. As can be seen in supplementary figure 1, where the UV-vis absorption spectra are displayed, a very high degree of crystallinity is thus obtained.

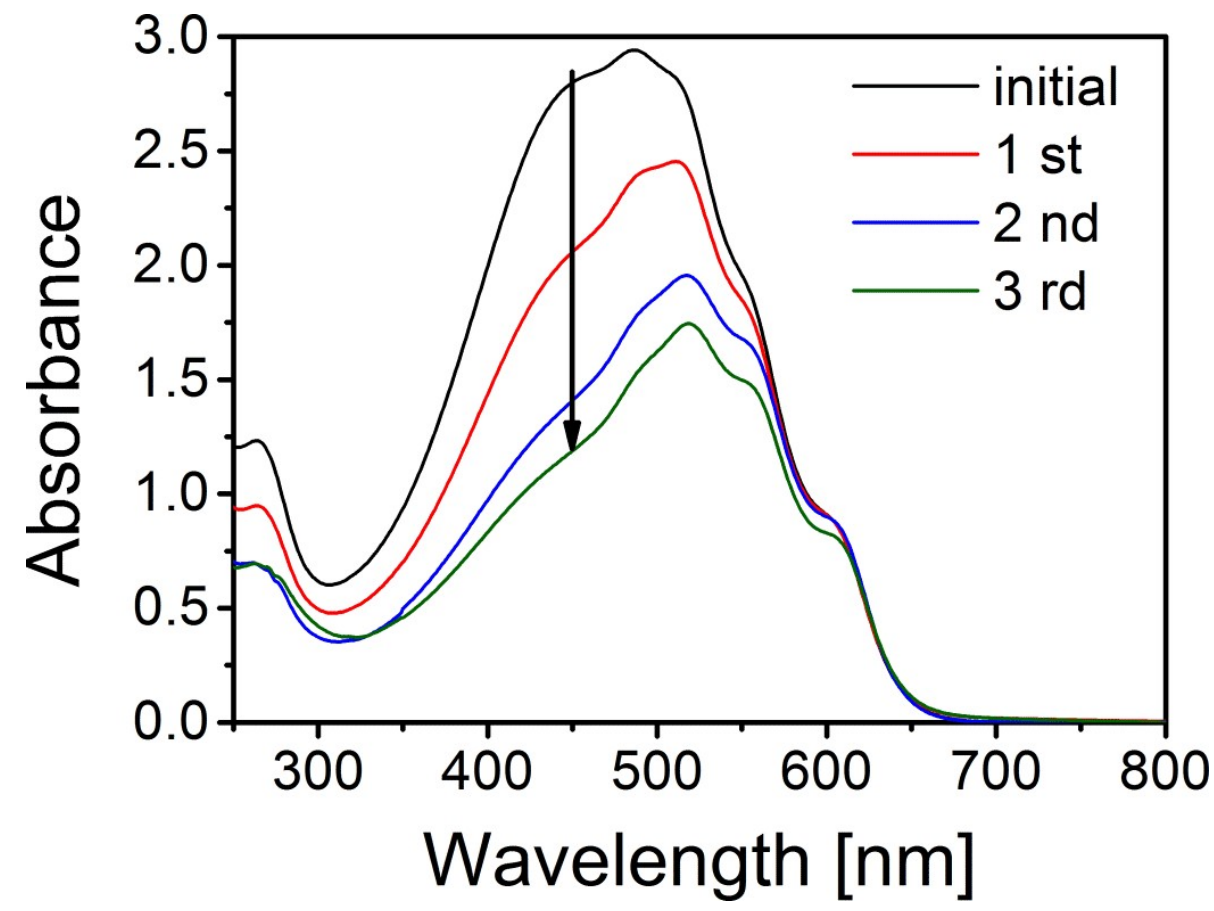

Supplementary Figure 1. UV-Vis absorbtion spectra after successive centrifugations of a P3BT/transdecahydronaphthalene suspension. The decrease of the maximum at $450 \mathrm{~nm}$ indicates gradual disappearance of the non-crystallizable chains. 


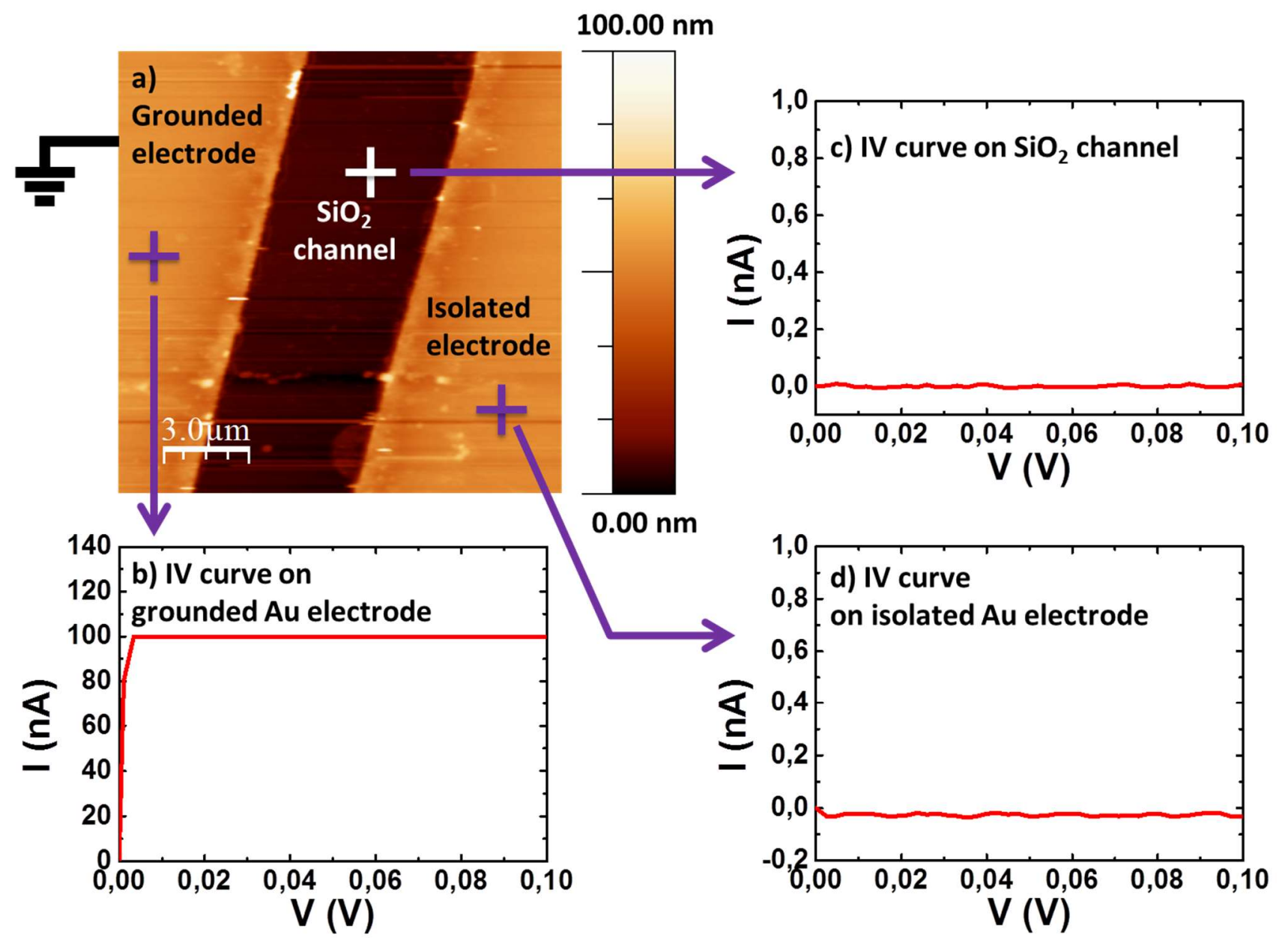

Supplementary Figure 2. IV curves on the different parts of the bare substrate. a) AFM topographic image of interdigitated Au electrodes acquired using PtSi tip in tapping mode; b) IV curves measured using the PtSi tip on the ground electrode show a linear relation with steep slope - characteristics of a conducting material; c) and d) IV curves show no current on the $\mathrm{SiO}_{2}$ channel and the isolated electrode respectively. 

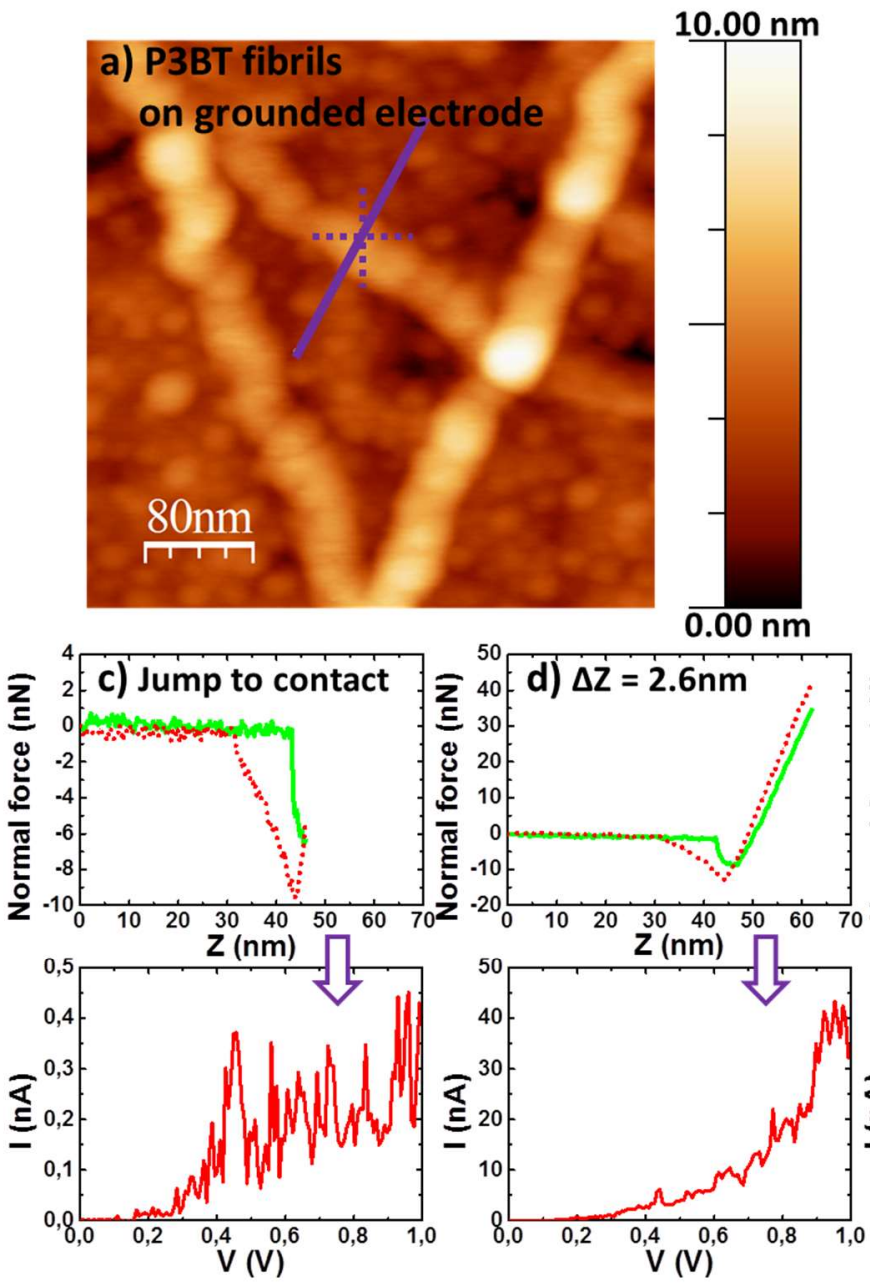
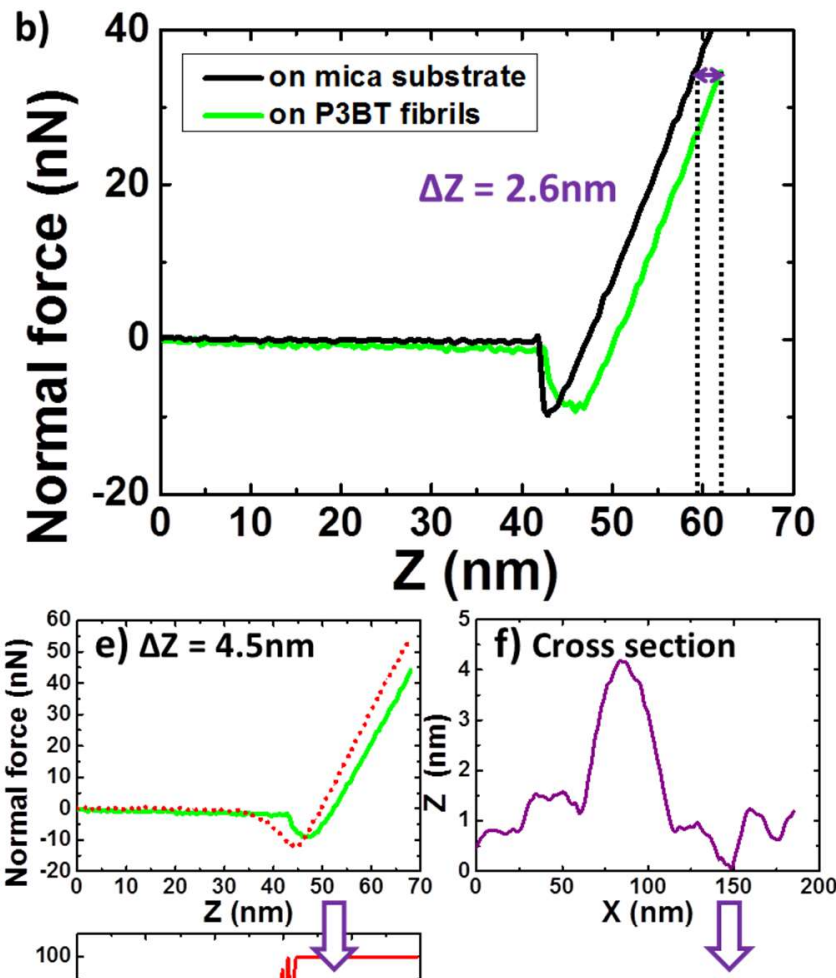

Fiber height $=4.2 \mathrm{~nm}$ in excellent agreement with $\Delta \mathrm{Z}=4.5 \mathrm{~nm}$

Supplementary Figure 3. Current-Voltage spectroscopy on P3BT fibrils at controlled indentation depth. a) Topography of P3BT fibrils on a grounded Au electrode; $b) \Delta Z$ calculation of P3BT fibril tip penetration by comparing FZ curves on mica; c-e) FZ curves at different tip depth positions $(\Delta Z)$ into the fiber (at the point located by the dotted purple cross) and the related I-V curves measured for each $\Delta Z$, confirming the semiconducting character of the P3BT fibrils (non linear $\mathrm{I}-\mathrm{V}$ curve); f) cross-section analysis of P3BT fibrils along the straight line in panel a. 


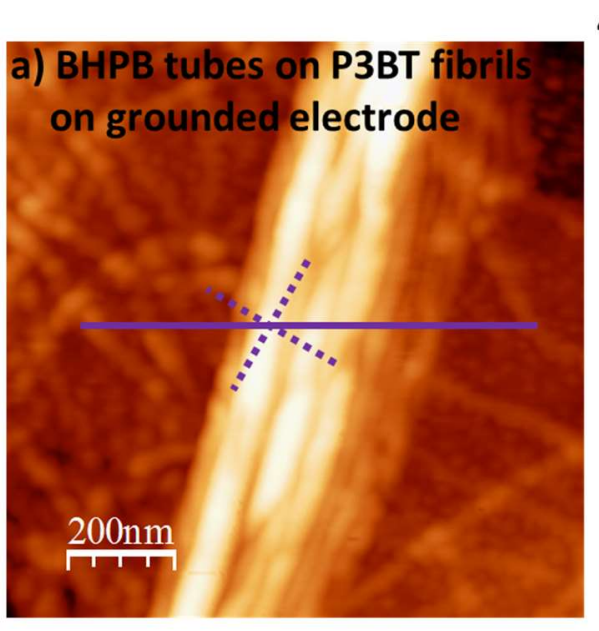

$45.00 \mathrm{~nm}$
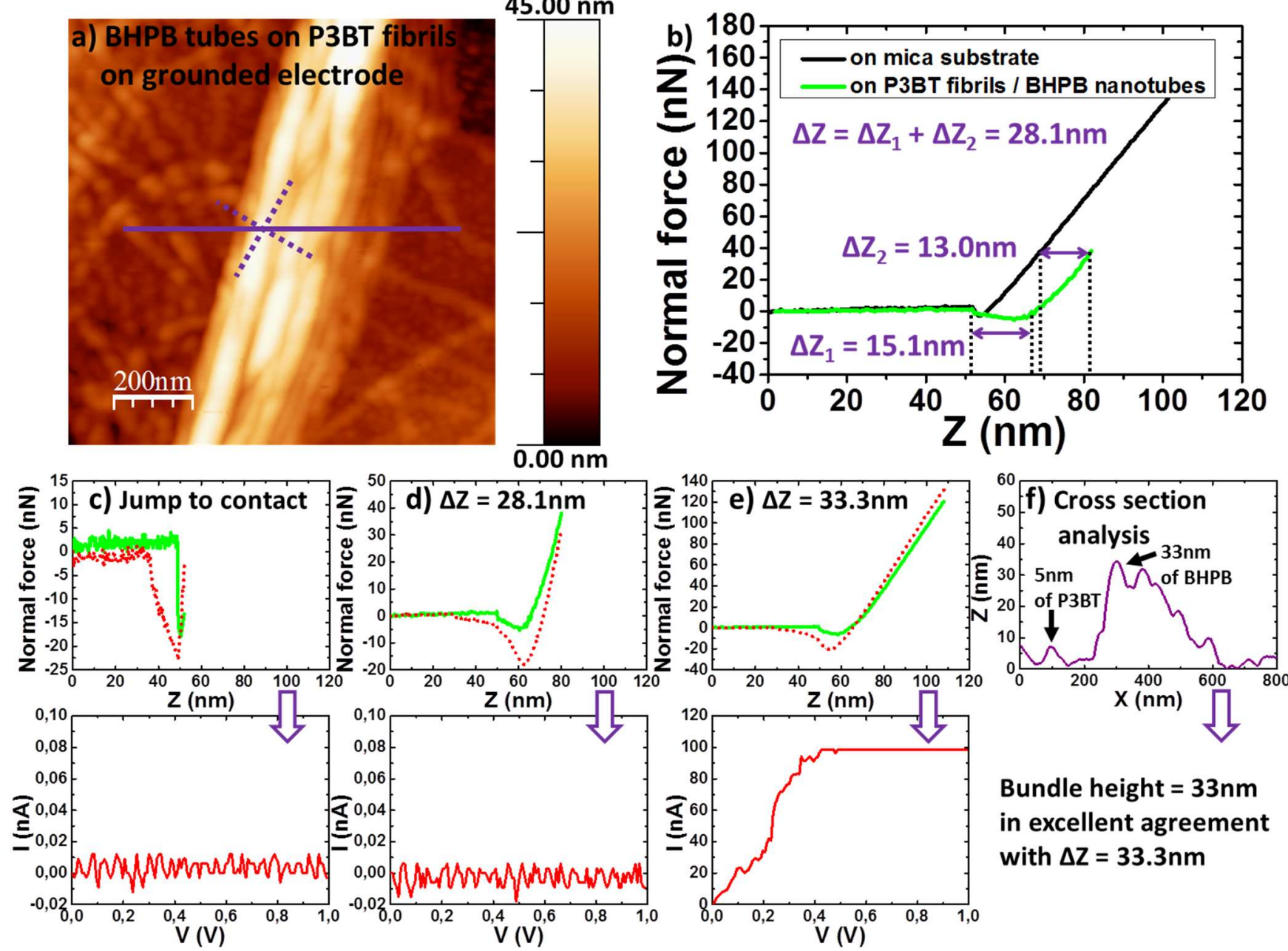

Bundle height $=33 \mathrm{~nm}$ in excellent agreement with $\Delta \mathrm{Z}=33.3 \mathrm{~nm}$

\section{Supplementary Figure 4. Current-Voltage spectroscopy on sequentially deposited BHPB-10 fibers on P3BT} fibrils at controlled indentation depth. a) Topography of BHPB-10 nanotubes on P3BT fibrils deposited on a grounded Au electrode; b) Calculation of tip penetration depth. The total tip penetration $(\Delta Z)$ here is obtained by the addition of the distance traveled by the tip through the spacing between fibrils, indicated by zero force from jump to contact until the point of linearly increasing load, $\left(\Delta Z_{1}\right)$, to the tip penetration distance into the fiber obtained by comparing FZ curves on mica $\left(\Delta Z_{2}\right)$; c-e) FZ curves at different tip depth positions $(\Delta Z)$ into the material (at the point located by the dotted purple cross) and the related I-V curves measured for each $\Delta Z$, confirming the fact that P3BT and BHPB-10 form separated entities under these conditions; f) cross-section analysis of P3BT fibrils and BHPB-10 nanotubes along the straight line in panel a. 
Supplementary Figure 5.
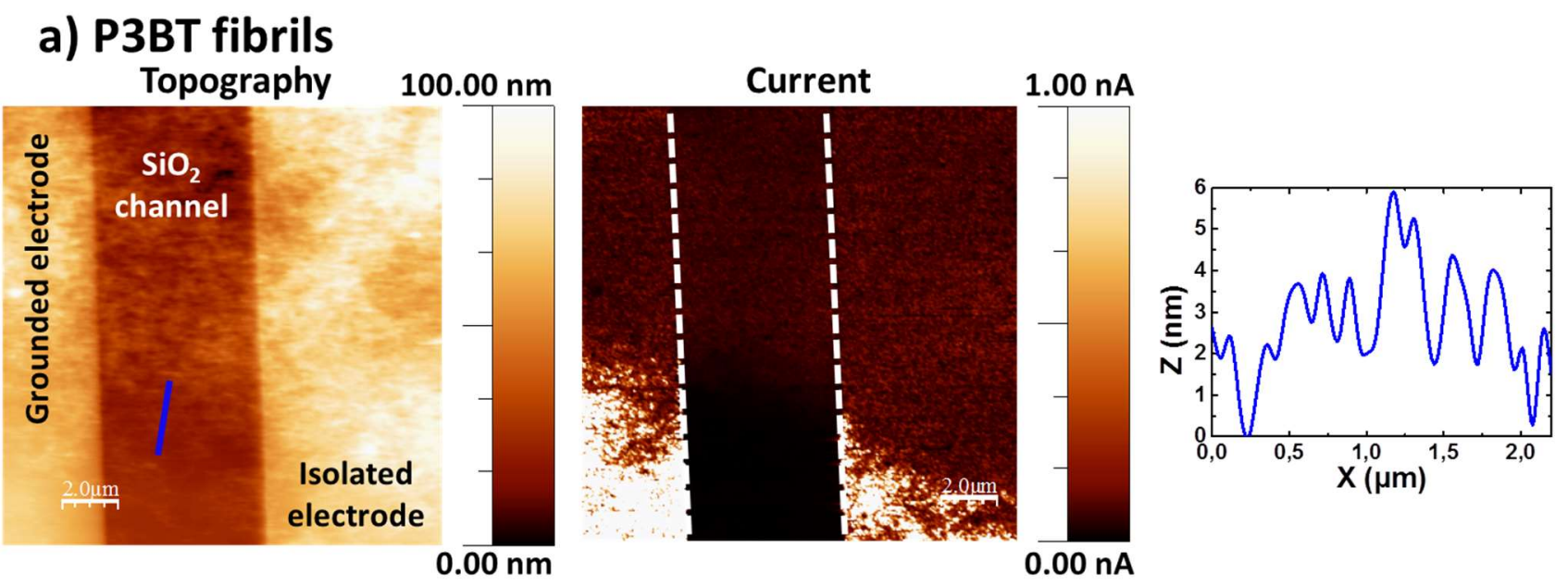

\section{b) BHPB nanotubes}
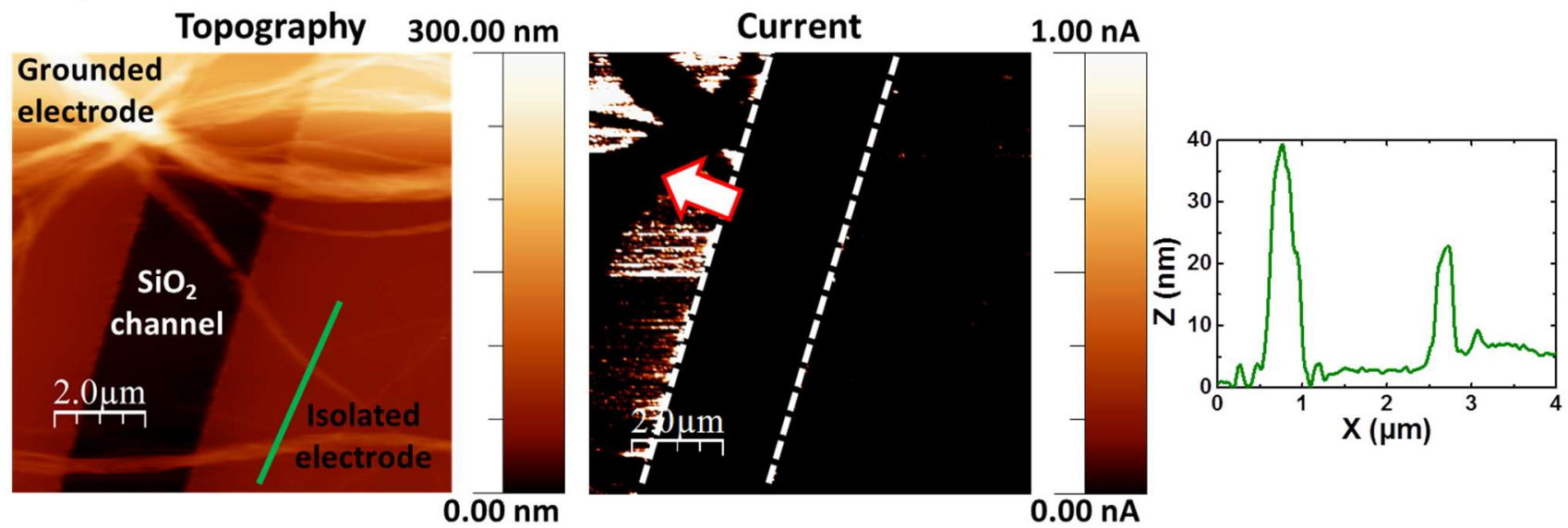

Supplementary Figure 5. AFM topography and C-AFM current mapping (see Fig. 3a for the experiment principle):
a) on P3BT fibrils: the current flows between both electrodes and channel indicating the conducting nature of P3BT;
b) on BHPB-10 nanotubes: the current flows only at the grounded electrode. No current appears in the channel and the isolated electrode. Red arrow indicates dark areas that correspond to BHPB-10 nanotubes on the ground electrode where no current is recorded due to the insulating nature of BHPB-10.
Cross-section analysis along the straight line in the topography panel are shown besides each image. 


\section{Neutron experiments}

A better fit for the P3BT fibrils is obtained by considering a rectangular cross-section. Considering a cylinder cross-section fits the low q domain but fails at larger q.

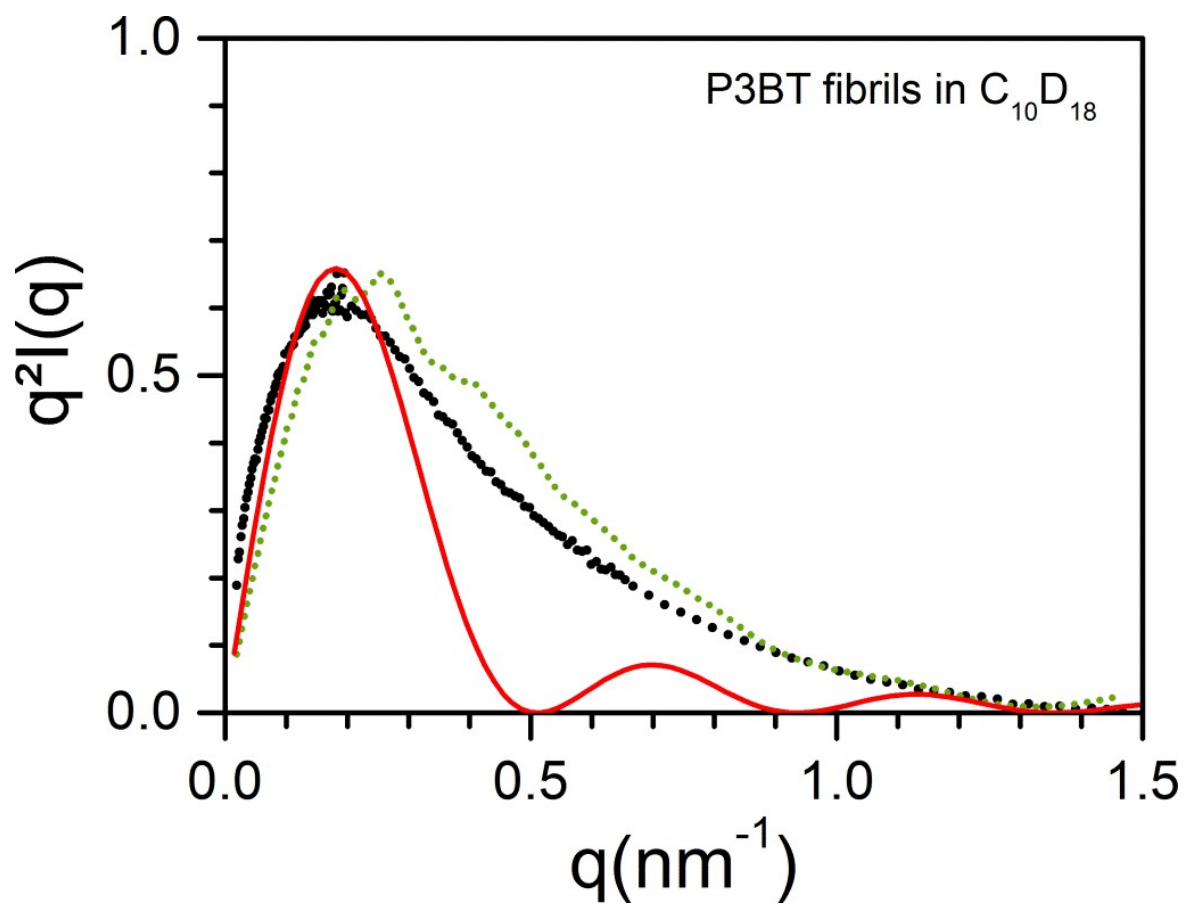

Supplementary Figure 6. Scattering by P3BT fibrils in deuterated trans-decahydronaphthalene. Comparison between a fit with a solid cylinder of radius $7.5 \mathrm{~nm}$ (red curve) and a ribbon of rectangular cross-section a= 4 $\mathrm{nm}$ and $\mathrm{b}=14 \mathrm{~nm}$. Note that $7.5 \mathrm{~nm}$ is the value of the half-diagonal of such a rectangle.

\section{Neutron contrast}

The contrast of P3BT with respect to deuterated trans-decahydronaphthalene (TDecaD) is:

$$
A_{P 3 B T}^{2}=\left(B_{P 3 B T}-\frac{v_{P 3 B T}}{V_{T D e c a D}} B_{T D e c a D}\right)^{2}
$$

Where $v$ with the appropriate subscripts are the molar volume of deuterated trans-decahydronaphthalene and P3BT respectively,

The scattering amplitude are calculated by summing the scattering amplitudes $a_{i}$ of all the atoms composing the molecules, namely

$$
\begin{aligned}
& B_{P 3 B T}=8 a_{C}+8 a_{H}+a_{S} \\
& B_{\text {TdecaD }}=10 a_{C}+18 a_{D}
\end{aligned}
$$

Similarly, for BHPB-10 


$$
A_{B H P B-}^{2}=\left(B_{B H P B-10}-\frac{v_{B H P B-10}}{V_{T D e c a D}} B_{T D e c a D}\right)^{2}
$$

With

$$
B_{B H P B-}=39 a_{C}+68 a_{H}+6 a_{O}+2 a_{N}
$$

$a_{c}=0.662, a_{H}=-0.375, a_{D}=0.670, a_{0}=0.575, a_{N}=0.94, a_{S}=0.285$

$\mathrm{d}_{\mathrm{P} 3 \mathrm{~B} T}=1.24 \mathrm{~g} / \mathrm{cm}^{3}, \mathrm{~d}_{\mathrm{BHPB}-10}=1.1 \mathrm{~g} / \mathrm{cm}^{3}$

$$
\begin{gathered}
A_{P 3 B T}^{2}=113 \times 10^{-24} \mathrm{~cm}^{2} \\
A_{B H P B-10}^{2}=4453 \times 10^{-24} \mathrm{~cm}^{2}
\end{gathered}
$$

